\title{
Compensating for a lack of skill
}

Although it is nice to think that every person who works with animals has all the skills needed to accomplish his or her tasks, reality tells us otherwise. For instance, Jody Marshall, Dr. Julia Holland's only technician, was expert at gavage and most other basic techniques, but she always had trouble with intravenous (i.v.) injections into the tail veins of conscious mice. No matter how hard she tried or how much training she received, she simply could not master that particular skill. To compensate for this problem, Holland always wrote in her IACUC protocol that mice would be lightly anesthetized with isoflurane before receiving i.v. injections. Isoflurane was administered in a closed container via a cotton pledget that was soaked with the liquid. Once anesthetized, the mouse was removed from the jar and the i.v. injection was quickly made. This method met Holland's need for the injections to be made into the tail vein, and it met the IACUC's needs because the technique was done correctly.

When Holland and Marshall moved to Great Eastern University, however, things changed. The Great Eastern IACUC was not willing to accept a lack of adequate skill as a reason to administer an anesthetic. Holland asked the IACUC to reconsider its vote, and at the next committee meeting, she presented her justification. First, she described the numerous honest attempts Marshall had made to become proficient at i.v. injections. Next, she emphasized the cost of the drugs she used, telling the committee that although the drugs were not irritating if they were injected incorrectly, at nearly $\$ 600$ per $0.2 \mathrm{ml}$, and with at least three injections needed over the course of an experiment, she could not afford to have mistakes. Her final argument was based on her experience at her former institution. There, the IACUC routinely approved using isoflurane because its use was brief and considered safe. In fact, Holland said she had never lost an animal from an anesthetic overdose.

After Holland left the room, the IACUC discussed her protocol. Some members agreed with Holland, saying that the explanation she presented was logical and sufficient. Others, including the veterinarian and an anesthesiologist, disagreed, saying that general anesthesia is not innocuous and that eventually the averages would catch up with Marshall. They were convinced that in due course she would lose mice as a result of the anesthesia. Intravenous injection is a basic research procedure, they said, and a technician with so many other skills could and should learn this one as well. What is your opinion? Should the IACUC approve Holland's study or take a different approach?

\section{RESPONSE}

\section{Poor methodology}

\section{Mary V. Shaw, MS, LVT}

It may be common for a technician to have problems acquiring all the necessary skills to do his job, but attitude goes a long way towards mastery. From time to time, I have also struggled to acquire new skills, especially those that require visual acuity and fine motor skills. But I found that 'difficult' is not 'impossible'. No mention is made of Marshall's attitude, but because Holland is willing to compensate for the lack of skill, I assume that Marshall has no real need to develop a 'can-do' attitude. Practice makes perfect for anyone with a willing attitude, a drive to excel and persistence to keep practicing until proficient.
I agree with the IACUC members who did not accept Holland's justifications for anesthesia. In addition to the concerns of the veterinarian and anesthesiologist, I have other problems with her protocol. There should be no variable introduced that could interfere with the welfare of the animals. Anesthesia, in this case, is an unnecessary variable that creates real risk to the health of the animals. Another consideration is that volatile gas anesthesia is processed through the liver and kidneys to some extent, however minute. That's another variable in the mix. The method in which anesthesia is being used in this case is also unacceptable. Isoflurane is extremely volatile and requires a vaporizer to ensure that it never vaporizes above $5 \%$. Using a closed container and a soaked pledget, Holland would have no idea how much isoflurane is administered to each animal. When administered this way, the amount of isoflurane would certainly be inconsis- tent and, quite likely, excessive. Holland and Marshall have been extremely lucky if they have not lost animals in the past using this method. The inconsistent dosing and lack of measurement of each animal's exposure introduce another variable to the study.

I have several suggestions. Holland should hire another technician who is proficient in tail vein injections to carry out these procedures. Perhaps she can trade Marshall's services to another researcher for the duration of this experiment. Or, she can delay the project until Marshall establishes her proficiency. If her job is on the line, I would bet that Marshall will become proficient. Given the cost of the drug, and the risk of a mistake during injection, proficiency in this technique is key to the success of the experiment. Because Marshall can administer i.v. injections to anesthetized animals, I believe she will ultimately succeed in doing so without anesthesia. 